\section{Contribuição dos agentes indígenas de saúde na atenção diferenciada à saúde dos povos indígenas brasileiros}

\author{
The contribution of indigenous community \\ health workers to special healthcare for \\ Brazilian indigenous peoples
}

Eliana Elisabeth Diehl 1,2

Esther Jean Langdon 3,4

Raquel Paiva Dias-Scopel 5
${ }^{1}$ Centro de Ciências da Saúde, Universidade Federal de Santa Catarina, Florianópolis, Brasil.

2 Programa de Pósgraduação em Assistência Farmacêutica, Universidade Federal de Santa Catarina, Florianópolis, Brasil.

${ }^{3}$ Centro de Filosofia e Ciências Humanas, Universidade Federal de Santa Catarina, Florianópolis, Brasil. 4 Programa de Pósgraduação em Antropologia Social, Universidade Federal de Santa Catarina, Florianópolis, Brasil. 5 Instituto Leônidas e Maria Deane, Fundação Oswaldo

Cruz, Manaus, Brasil.

Correspondência E. E. Diehl Departamento de Ciências Farmacêuticas, Centro de Ciências da Saúde, Universidade Federal de Santa Catarina.

Campus Universitário, Bairro Trindade, Florianópolis, SC 88040-900, Brasil. elianadiehl@hotmail.com

\section{Abstract}

Indigenous community health workers are part of a strategy developed by Brazil in the last two decades to promote a special healthcare model for indigenous peoples. Their role is designed to deal with various aspects of the special health policy, including the link between the heath team and the community and mediation between scientific and indigenous medical knowledge. Despite a significant increase in the number of indigenous community health workers in recent years, an evaluation of their responsibilities and contributions to the success of special care had not been conducted previously. This article, based on a literature review and original research by the authors, analyzes the role of the indigenous community health workers vis-à-vis their training and participation in health teams in different contexts in Brazil. Considering the importance assigned to the role of indigenous community health workers, this analysis reveals various ambiguities and contradictions that hinder both their performance and their potential contribution to the special health services.

Health of Indigenous Peoples; Community Health Workers; Indigenous Health Services

\section{Introdução}

A noção de atenção diferenciada para os povos indígenas do Brasil construiu-se segundo princípios e modelos propostos em diferentes documentos 1,2,3, iniciando-se com a la Conferência Nacional de Proteção à Saúde do Índio, em 1986, e culminando no estabelecimento do Subsistema de Atenção à Saúde Indígena, em 1999. Uma das estratégias desenvolvidas para alcançar seus objetivos foi a institucionalização do agente indígena de saúde (AIS) como parte das equipes que prestam serviços de atenção primária nas aldeias. A institucionalização do AIS visa atender vários aspectos abordados nesses documentos, como a criação de cargos assalariados para membros da comunidade. O papel do AIS é considerado central na realização do princípio da atenção diferenciada, ou seja, na oferta de serviços de saúde sensíveis ao pluralismo e à diversidade cultural, incorporando o direito da comunidade de participar, individual ou coletivamente, em seu planejamento, execução e avaliação. Formar e capacitar AIS, segundo a Política Nacional de Atenção à Saúde dos Povos Indígenas (PNASPI) 4, é fundamental para sua atuação na atenção primária. Porém, a metodologia de ensino e o conteúdo programático dos cursos não dão conta das especificidades inerentes à saúde indígena, pois o conhecimento biomédico é valorizado nesses cursos, reproduzindo as 
relações assimétricas entre os povos indígenas e a sociedade ocidental.

Por intermédio de nossas pesquisas sobre o papel do AIS em Santa Catarina e outras realizadas no Brasil, este artigo examina, após 10 anos de sua institucionalização no Subsistema, a implantação, formação e participação dos AIS nas Equipes Multidisciplinares de Atenção Básica à Saúde Indígena (EMSI) e sua contribuição para a atenção diferenciada.

A revisão dos trabalhos considerou grupos de pesquisa ou pesquisadores que vêm acompanhando e publicando sobre a implantação de serviços de atenção à saúde indígena nas últimas quatro décadas, em Terras Indígenas e regiões do país que historicamente têm sido objeto de estudos etnográficos, com destaque para os recursos humanos. Ainda, consultou-se a biblioteca SciELO, o banco de teses da CAPES e sítios eletrônicos de organismos nacionais e internacionais para consulta de documentos oficiais. A participação em bancas examinadoras e em eventos sobre a temática também forneceram dados.

\section{Antecedentes}

A concepção do papel do AIS norteou-se na proposta para o agente comunitário de saúde (ACS), lançada há mais de três décadas e expressa na Declaração da Alma-Ata ${ }^{1}$. No Brasil, os ACS surgem oficialmente em 1991, e em 1992 integram o Programa de Agentes Comunitários de Saúde (PACS), incorporados também nas equipes do Programa Saúde da Família (PSF) a partir de 1994. Foram reconhecidos como profissionais da saúde em 20025 e submetidos ao regime jurídico da Consolidação das Leis do Trabalho (CLT) em 2006 6 . A profissão de ACS caracteriza-se pela prevenção de doenças e promoção da saúde, mediante ações domiciliares ou comunitárias, individuais ou coletivas, desenvolvidas conforme diretrizes do Sistema Único de Saúde (SUS) e sob supervisão do gestor local. Para Morosini 7 (p. 17), com a legislação e a criação da Secretaria de Gestão do Trabalho e da Educação na Saúde em 2003, “as questões da precariedade da formação, bem como da precariedade dos vínculos dos ACS passaram a se expressar na agenda do Ministério da Saúde de maneira central".

A 1ạ Conferência Nacional de Proteção à Saúde do Índio objetivou estabelecer os princípios da organização da saúde indígena, introduzindo o conceito de atenção diferenciada, sugerindo a formação em saúde de indígenas nas próprias aldeias e a observância de critérios e definições das comunidades para indicação e remuneração dos agentes 8 .
A 2a Conferência Nacional de Saúde Indígena (1993) recomendou ao Ministério da Saúde o reconhecimento da categoria profissional de AIS. Em 1996, a Coordenação de Saúde do Índio (COSAI) da Fundação Nacional de Saúde (FUNASA) outorgou ao AIS o papel-chave na mediação dos serviços de saúde, como ator central para a promoção dos princípios de respeito e articulação com as tradições indígenas 9, indicando ainda que a formação incluísse outras temáticas além das noções básicas de biomedicina e de enfermagem. Contudo, a COSAI não teve condições financeiras nem organizacionais para implantar um programa amplo e eficaz de formação e remuneração dos AIS, e o PACS assumiu maior responsabilidade nas contratações 10 .

Na prática, desde o final da década de 1970 os AIS estavam sendo treinados e incluídos nos serviços de atenção primária prestada por algumas universidades, organizações não governamentais (ONG) indígenas e não indígenas e grupos ligados à Igreja católica. Destacam-se as experiências do Conselho Indigenista Missionário 11, do Projeto Xingu (Mato Grosso) 12,13,14,15, do Rio Envira (Acre) 16, da região do Rio Negro (Amazonas) 17,18, dos Tikúna (Amazonas) 10,19 e de Santa Catarina 20. Em muitos casos, foram introduzidos para fins de tradução e ampliação da atenção primária às populações isoladas, de difícil acesso e sem domínio da língua portuguesa. Suas tarefas, responsabilidades e remuneração variaram dependendo do contexto específico e do provedor de serviços básicos. Na segunda parte dos anos 1990, foram oferecidos cursos de capacitação aos AIS do Rio Negro 21, com base numa abordagem educacional mais dialógica, reconhecendo que os indígenas ativamente reformulam e ressignificam os conhecimentos apresentados nos cursos 17. Nesse caso, muitos que realizaram os cursos foram subsequentemente contratados pelo PACS. Já no início de 2000, Yanomámi foram treinados e contratados como microscopistas no combate à malária 22. No sul do país, a Pastoral da Criança organizou cursos para capacitação em primeiros socorros e fitoterapia, entretanto poucos indígenas foram absorvidos pelo PACS 23 , como ocorreu em outras regiões.

Alguns autores publicaram reflexões e avaliações sobre os projetos Rio Envira, Xingu e Alto Rio Negro, nos quais se verificam certas características que os distinguem daqueles que vinham sendo implementados pela FUNASA após 1999 $12,13,17,21,24$. Tais cursos foram caracterizados pelo acompanhamento antropológico, procurando adequar os projetos às especificidades culturais. As atividades realizadas nas estratégias pedagógicas e nas atribuições do papel do AIS foram 
desenvolvidas em função de cada contexto e da motivação para resolver os problemas locais de doenças, distribuindo os serviços de saúde de maneira sensível à realidade cultural na qual a equipe se encontrava. Assim, os cursos de formação dos AIS foram desenhados conforme especificidades locais, com grupos étnicos particulares, visando à adaptação das estratégias pedagógicas a essas realidades, tendo ainda uma abordagem biomédica e tecnicista.

\section{FUNASA e os agentes indígenas de saúde}

A institucionalização do AIS se tornou possível a partir de 1999 com a criação dos Distritos Sanitários Especiais Indígenas (DSEI) e do aumento no orçamento para os serviços de saúde indígena 25 , sob responsabilidade do Departamento de Saúde Indígena (DESAI) da FUNASA. As EMSI são estabelecidas como peça fundamental na atenção primária oferecida nas Terras Indígenas, e o AIS faz parte dessa equipe junto com médicos, dentistas, enfermeiros, técnicos de enfermagem e outros profissionais 26 .

Objetivando normatizar a implantação do Programa de AIS como parte do processo de construção dos DSEI, em 1999 a FUNASA relatou existirem no país cerca de 2.000 agentes no final dos anos 1990, e mais de $70 \%$ deles trabalhavam sem acompanhamento ou supervisão 27 . Também apontou a necessidade da capacitação contínua, sob responsabilidade do instrutor-supervisor da equipe de saúde dos Distritos, com a participação e colaboração de outros profissionais. Recomendou que o reconhecimento da profissão de AIS fosse garantido, citando a Lei de Diretrizes e Bases da Educação Nacional (LDB) 28.

A 3a Conferência Nacional de Saúde Indígena (2001) reafirmou a importância do papel do AIS e aprofundou as demandas para formação, reconhecimento e inserção social dos agentes, como: aumento do número de AIS por comunidade e EMSI, necessidade de formação básica para todos, garantia do processo de capacitação contínua e diferenciada, acompanhamento técnico/pedagógico e administrativo no processo de formação e contratação pela CLT 29.

A importância do AIS no princípio da atenção diferenciada é reafirmada na PNASPI ${ }^{4}$. A atenção diferenciada contempla noções de respeito às concepções, valores e práticas em saúde de cada povo e de articulação entre os saberes indígenas e biomédicos, e o agente indígena é o mediador entre esses saberes, assim como entre a comunidade e os membros da equipe 4 . O AIS deve ser um membro da comunidade e ideal- mente eleito por ela, atuando na atenção primária. A capacitação e a supervisão do AIS são fundamentais para consolidar seu papel e para o êxito de sua atuação.

Pela PNASPI, e com recursos financeiros adequados, o número de AIS contratados mais que dobrou entre 2000 e 2006, junto com o aumento das EMSI. Dados apontam uma diminuição em 2007 (Tabela 1), mas, na falta de informações sobre os últimos anos, é difícil interpretar essa retração.

Apesar do aumento do número de AIS contratados, não se pode definir de forma homogênea e estática o seu papel 13,30,31,32 nem definir que a problemática da formação e supervisão tenha sido resolvida. A diversidade geográfica e o grau de contato com a sociedade envolvente permanecem espelhando a heterogeneidade de papéis. No caso dos grupos mais isolados, alguns seguem realizando cuidados primários e servindo de intérpretes. Nas comunidades em contato contínuo com a sociedade envolvente, os AIS trabalham como assistentes dos profissionais das EMSI com tarefas limitadas e mal definidas, como é o caso de Santa Catarina 33. A variação do grau de escolarização do AIS também contribui para a heterogeneidade de suas ações e papéis, pois enquanto alguns possuem o ensino básico ou médio, outros nem têm formação escolar.

A PNASPI ${ }^{4}$ ressalta a prioridade de cursos de capacitação dos AIS. Desde então, foram desenvolvidos modelos pela FUNASA no nível nacional, orientando a formação às questões técnicas sobre problemas comuns de saúde. No entanto, os dados sobre o número de AIS capacitados ao longo dos anos são poucos e contraditórios. $\mathrm{Na}$ análise sobre a implantação do Subsistema e as atividades da FUNASA, Garnelo et al. 34 reconhecem a ênfase especial dada à formação dos AIS, mas simultaneamente observam dificuldades na

Tabela 1

Número de agentes indígenas de saúde (AIS) após a criação dos Distritos Sanitários Especiais Indígenas.

\begin{tabular}{ll}
\hline Período & AIS \\
\hline $2000 *$ & 2.284 \\
$2002 *$ & 2.732 \\
$2006 * *$ & 5.106 \\
$2007 * * *$ & 3.833 \\
\hline
\end{tabular}

Fonte: * Fundação Nacional de Saúde 53; ** Fundação

Nacional de Saúde 54; *** Institute of Development Studies/

Saúde Sem Limites/Centro Brasileiro de Análise

e Planejamento 55 . 
provisão dos cursos de capacitação para os AIS e os demais profissionais.

Apesar da multiplicidade de contextos e atividades dos AIS nos DSEI, a revisão das pesquisas indica certas tendências gerais, dificuldades e avanços na institucionalização do papel do agente na última década, que podem ser resumidos em quatro aspectos: alta rotatividade e suas implicações; ambiguidade do papel; relacionamento com os membros das equipes de saúde; e formação e capacitação.

\section{Alta rotatividade e suas implicações}

A alta rotatividade nas EMSI é uma característica tanto entre os profissionais não indígenas quanto entre os AIS, e é um problema desde o início da implantação dos DSEI ${ }^{34}$, resultando em interrupções no atendimento, mesmo nas regiões relativamente acessíveis. A irregularidade dos serviços resulta dos problemas de organização e contratação, entre eles os convênios realizados pela FUNASA com as ONG 34 , renovados anualmente e que ocasionam rupturas de pagamentos e insegurança de emprego. A grande rotatividade dos profissionais traz repercussões diversas para a qualidade da prestação dos serviços, impactando nas práticas de controle das informações e dados sobre saúde, refletindo na descontinuidade desses dados 35 e na capacitação, já que ela ainda é muito irregular 19,36. Também influi negativamente na supervisão dos AIS e nas suas relações profissionais.

Alguns fatores causadores da rotatividade dos AIS são os mesmos que acarretam a alta rotatividade dos demais profissionais da EMSI: o tipo de vínculo empregatício definido pelas contratações anuais e as divergências salariais. Entre os Xokléng, observamos que a alta rotatividade dos AIS decorreu dos intervalos entre as renovações dos convênios da FUNASA com a ONG, que provocavam períodos de trabalho sem salário, desestimulando a permanência no cargo 31,33. A divergência entre os salários dos profissionais também desestimula a permanência no emprego, particularmente no caso de aldeias isoladas, onde o AIS é o único membro permanente da EMSI, realizando atividades de outros profissionais mais bem remunerados. Uma solução para a insatisfação dessas condições de trabalho tem sido a busca de formação técnica, a exemplo de Santa Catarina, como veremos adiante.

A revisão das etnografias aponta que os processos de indicação dos indígenas ao cargo de AIS são fatores adicionais na rotatividade deles e que precisam ser entendidos e avaliados dentro das particularidades socioculturais de cada povo e do contexto político-partidário regional.

Na indicação dos AIS, sobressaem-se as dinâmicas da organização social e dos processos políticos inerentes a cada grupo indígena como fatores constituintes dos critérios de legitimidade. Entre os Baniwa, por exemplo, as indicações para os cargos de agentes seguem as dinâmicas sociocosmológica e política das hierarquias internas, e também os critérios inerentes à legitimação de cargos de liderança e prestígio, como domínio da língua portuguesa e desenvoltura nas interlocuções com os brancos 25,36. Erthal 19 detalha como a escolha de AIS é feita entre as afiliações políticas das lideranças Tikúna e o surgimento de conflitos como consequência da criação do cargo assalariado. Rocha 37 indica que os não indígenas da EMSI reclamaram que os AIS, em uma aldeia Kaingáng de Santa Catarina, foram selecionados segundo alianças de parentesco e não segundo suas capacidades.

As organizações sociais e políticas dos indígenas que implicam a escolha dos AIS e sua eventual demissão ou troca não devem ser vistas de modo negativo, pois são esses mesmos arranjos que constituem os parâmetros de controle sobre as ações dos agentes. O Projeto Xingu é particularmente ilustrativo da importância da organização social e dos processos políticos no êxito do desempenho dos AIS. Inicialmente, a equipe médica escolheu os AIS segundo seus critérios. Porém, tal estratégia causava falta de aceitação da comunidade e insegurança dos agentes. Subsequentemente, o processo de seleção dos AIS foi negociado nas aldeias e as escolhas seguiram as linhas de poder político e as linhagens de parentesco das aldeias, resultando em mais segurança dos AIS e um melhor relacionamento com as comunidades 13 .

Já no DSEI Rio Negro-Amazonas, critérios técnicos passaram a compor a exigência de pré-requisitos para a indicação dos AIS, como a escolaridade formal 36. De modo semelhante, entre os Xokléng, a equipe do polo base, a ONG conveniada e o DSEI-Interior Sul/Santa Catarina realizaram, em 2003, um processo seletivo por meio de prova, buscando contornar os critérios e processos sociopolíticos Xokléng de legitimação dos cargos de liderança e de AIS condizentes com as relações de poder e de parentesco 31,33. Em ambos os contextos, as consequências dos critérios técnicos usados para controlar o processo de indicação dos AIS foram a insegurança deles e a saída de alguns que não se adequavam aos requisitos.

Os arranjos político-partidários regionais são fatores que também impactam na rotatividade da EMSI. No contexto de Santa Catarina, 
repercutiram na composição da EMSI contratada pelo PSF vinculado ao município. Na Terra Indígena Xapecó ${ }^{33}$, entre maio e julho de 2004, havia dois grupos de AIS que se distinguiam pela contratação via ONG indígena (sete agentes) e via um dos municípios (nove AIS). Durante a pesquisa, realizada em ano eleitoral, os AIS manifestaram temor quanto à perda de emprego caso fosse eleito o candidato da oposição, o que de fato aconteceu. Em janeiro de 2007, observou-se que nas aldeias localizadas neste município havia 12 AIS, todos contratados pelo PSF, sendo que, dos 16 que trabalhavam em 2004, permaneciam no cargo menos da metade; em fevereiro de 2008, dos nove AIS contratados pelo PSF, somente dois deles trabalhavam desde 2004. Apesar de a PNASPI ${ }^{4}$ prever a contratação dos AIS segundo a indicação comunitária, o que se vê na prática dos DSEI é uma releitura das diretrizes, que põe em suspensão os critérios étnicos dos povos indígenas em favor de critérios técnicos baseados no domínio de conhecimentos ocidentais.

\section{Ambiguidade do papel}

Várias ações de saúde vêm sendo atribuídas aos AIS, abrangendo atividades relacionadas ao acompanhamento de pacientes, atendimento, primeiros socorros, prevenção e promoção da saúde ${ }^{38}$. Na prática, as ações realizadas pelos AIS são diversas e o contexto específico as demarca sobremaneira. Dois aspectos influenciam, em particular, a natureza das responsabilidades dos AIS: o grau de inserção do grupo na sociedade envolvente e a compreensão que a EMSI tem dos princípios da atenção diferenciada. O êxito em oferecer serviços que respeitem e se articulem com as práticas tradicionais depende muito do compromisso e dos esforços para capacitar todos os membros da EMSI, inclusive os AIS, para a atenção diferenciada.

No contexto de indígenas vivendo em região isolada (onde muitos não falam português), encontramos frequentemente uma delimitação mais clara do papel do AIS, como tem sido o caso do Xingu 13, do Rio Negro 21 e da área Yanomámi 22. Nessas regiões, o AIS tem um papel importante como intérprete e executor de serviços ampliados, realizando tarefas específicas como atendimento às doenças mais frequentes e administração de primeiros socorros na ausência da equipe. Todavia, nos contextos em que o grupo está em contato regular com os não indígenas e em maior dependência do mercado capitalista, as atribuições do papel do AIS tendem a ser mal definidas, resultando em ambi- guidades que tornam esse papel frustrante para todos os envolvidos.

Em Santa Catarina, as atividades delegadas aos AIS são amplas e imprecisas, gerando insegurança nos agentes 33 . Oficialmente, elas se agrupam em: (1) visitas domiciliares; (2) educação e orientação sobre cuidados de saúde e informações sobre o sistema de saúde e seu funcionamento, incluindo os programas especiais de saúde; (3) preenchimento de formulários; e (4) participação nas atividades de controle social. Os AIS indicaram que tais funções foram repetidamente focos de tensão e frustração. A lógica das visitas domiciliares se chocou com a lógica social e política das comunidades, gerando mau entendimento e o não cumprimento do objetivo formal da visita (o AIS passava nas casas para pedir a assinatura do chefe da família, sem realizar a entrevista completa exigida pelo serviço). Essas comunidades são organizadas mediante as redes de parentesco e práticas sociais que mantêm as pessoas a par dos acontecimentos, tornando supérflua a visita como recurso de obtenção de informações sobre a saúde da família, visto que o AIS é integrante da rede de comunicação. Ademais, como demonstrado em outras pesquisas 25,39 , se por um lado as redes sociais indígenas locais implicam sociabilidade, parentesco e relações políticas, por outro lado o cumprimento das funções e tarefas, como a visita a todas as casas, exige que o AIS opere independentemente desses processos locais.

A organização hierárquica das equipes delega os cuidados de atenção primária ao auxiliar ou técnico de enfermagem, e não aos agentes. Na Terra Indígena Xapecó, por exemplo, os AIS capacitados para algumas práticas de atenção básica reclamaram que essas tarefas foram proibidas, pois eram de responsabilidade do auxiliar de enfermagem. Da mesma maneira, o acompanhamento do crescimento das crianças também foi retirado de sua responsabilidade. Como em outros contextos da saúde indígena, o papel do AIS se tornou restrito à distribuição de medicamentos e de avisos relacionados às ações e serviços de saúde, como dia de vacinação, de exame ou de consulta especializada 30,31,33,35,39,40,41.

Entretanto, importa reconhecer que há casos em que o AIS contribui para o acompanhamento dos tratamentos de doenças crônicas e infectocontagiosas de longa duração, como observado na população Múra, em Autazes, Amazonas 42,43. A participação dos AIS revelou-se fundamental para o tratamento de alguns casos de tuberculose e hanseníase, especialmente naqueles em que a família não dispunha de recursos financeiros ou de tempo para deslocar-se até a cidade em busca do medicamento. 
Os AIS frequentemente se percebem alvos de preconceitos e que seu trabalho não recebe o reconhecimento dos membros das EMSI, nem dos profissionais nos serviços da rede de referência do SUS, aos quais eles muitas vezes servem de acompanhantes. Certamente, um fator que contribui para essa situação, além da natureza hierárquica implícita nos serviços médicos, é a falta de reconhecimento de seu papel como profissional.

Se por um lado os AIS declaram que suas relações com a equipe não são boas, eles também sentem que seu papel é mal entendido pela comunidade. Em 2004, os AIS Xokléng e Kaingáng reclamaram que ocupavam posições imersas em demandas contraditórias, pois as famílias esperavam que cumprissem atividades delegadas a outros membros da EMSI ("Elas acham que devemos ser médicos”), tais como medir febre e pressão arterial, recomendar e fornecer medicamentos e aplicar injeções 33 . Vianna \& Ott 44 também relatam um caso em que o AIS enfrentava demandas da comunidade que não fazem parte das atribuições do cargo.

Os AIS, da perspectiva das comunidades, são agentes representantes da prática biomédica, pois emergem no contexto da interação inter e intraétnica como atores que detêm um conhecimento específico e são capazes de garantir o acesso aos recursos materiais e simbólicos ofertados pelos serviços de saúde, como medicamentos 19,25,32. Essa perspectiva explica uma das razões para a seleção dos AIS como conselheiros de saúde nos níveis local e distrital. A emergência dos AIS como protagonistas em determinados cenários políticos de certa forma é reflexo do acesso privilegiado, embora não de maneira autônoma, aos saberes e práticas biomédicos 32,35. Por exemplo, os AIS Atikum (Pernambuco) participam ativamente como conselheiros locais de saúde, destacando nessa função, como representantes da prática biomédica, reivindicações dos pontos que dificultam a execução das ações 32. No outro extremo, no âmbito do DSEI Interior Sul e de seu Conselho Distrital, alguns AIS conselheiros expressaram ambiguidade sobre sua representatividade, por atuarem simultaneamente no Conselho como representantes dos usuários (e, portanto, vocalizadores das demandas comunitárias) e das instituições empregadoras 45 .

\section{Relacionamento com os membros das equipes de saúde}

As relações com os membros da EMSI raramente são positivas ou construídas em diálogo. Em parte, os profissionais nem sempre estão livres dos preconceitos que marcam as relações interétnicas já vigentes entre indígenas e não indígenas e, como já apontado, outros fatores que impedem uma relação dialógica são a organização hierárquica que permeia os serviços e a alta rotatividade dos membros da EMSI. Em Santa Catarina 33, os AIS com mais tempo de serviço relataram uma relação melhor no passado: "Hoje a equipe nem sabe quem é AIS”. Na sua avaliação sobre o exercício de habilidades específicas de outros profissionais da equipe afirmaram: "não sabe lidar com o índio, não conhece a nossa realidade". Por exemplo, os AIS da Terra Indígena Xapecó lembravam-se de um enfermeiro que, a seus olhos, demonstrou uma atitude de preconceito ao argumentar contra o uso do fogo de chão nas casas indígenas. Interpretando isso como uma acusação de que eles eram sujos, uma AIS comentou: "o índio não é sujo; todos nós temos roupa limpa no varal”.

A última afirmação não se trata de um caso isolado, mas de uma ideologia higienista e campanhista implícita na formação da FUNASA 46. Em uma reunião em 2004 sobre desnutrição entre os Guaraní-Kaiwá, a segunda autora desse artigo assistiu a uma palestra da nutricionista do polo base da FUNASA, que manifestou que as mães indígenas contribuem para a alta taxa de desnutrição, citando como causas a comida inconsistente, horários errados de alimentação e a falta de preocupação das mães. Wawzyniak 39 aponta que as equipes que visitam as comunidades ribeirinhas e indígenas no Rio Tapajós expressam a moralidade da biomedicina ao definirem sua tarefa como a de transformar os hábitos da comunidade, eliminando os tabus e superstições que resultam em problemas de higiene e nutrição.

A organização hierárquica das equipes desdobra-se nos procedimentos burocráticos exigidos aos AIS, tornando a relação entre eles e a EMSI ainda mais formal e assimétrica. Pesquisas apontam o preenchimento de inúmeros formulários como um fator que burocratiza seu trabalho e tende a servir muito mais como exercício de controle e fiscalização, substituindo o acompanhamento e a supervisão previstos 19,33,39,41,44. Os AIS manifestaram frustração com o número elevado de formulários a serem preenchidos, sem entenderem o objetivo de tais documentos 33 . Os AIS Xokléng reclamaram que os outros membros da EMSI não os escutavam e assim não conheciam os problemas: "pra que anotar tudo aquilo e mandar pra eles, eu acho que se eles olhassem aqueles papéis que a gente manda pra eles, eles estariam informados, saberiam qual é a nossa necessidade aqui" 40 (p. 111). 
As várias pesquisas indicam que a relação entre os profissionais indígenas e não indígenas da EMSI está marcada por ambiguidades que emergem da tensão entre um processo de trabalho hierárquico definido por atividades burocráticas e a dificuldade de compreensão e articulação entre maneiras diversas de tratar as questões relativas ao corpo, ao saudável, ao que é doença, ao puro/impuro, ao infortúnio, às dietas alimentares, às prescrições de hábitos etc.

\section{Formação e capacitação}

Como parte da institucionalização do AIS a partir de 1999, o processo de capacitação e supervisão tornou-se mais centralizado e burocrático, dependendo do DESAI/FUNASA em Brasília para sua definição, financiamento e cronograma, cabendo aos Distritos programar e executar os cursos. Se o aumento de contratações dos AIS significa um ponto positivo nos esforços da institucionalização de serviços diferenciados, a formação e a capacitação são talvez os aspectos mais fracos desses esforços. Por mais que a capacitação faça parte da Política Nacional desde a criação dos DSEI, as pesquisas revisadas demonstram que existem grandes lacunas em sua regularidade e continuidade e que a supervisão do trabalho do AIS, que contribuiria para o processo pedagógico em serviço, está ausente.

Bissera 22 relata uma interrupção na formação e participação dos indígenas como microscopistas e AIS em 2004, com a troca da ONG indígena pela Fundação Universidade de Brasília no convênio com a FUNASA. Quando a ONG assumiu o convênio em 1999, a malária era o problema mais crítico de saúde nesse grupo, priorizando a formação de microscopistas indígenas como parte de um programa de treinamento maior dos AIS. Eles foram formados em sua própria língua e submetiam-se a um exame oficial na Secretaria de Saúde do Estado de Roraima, possibilitando a ampliação do combate à doença e sua redução no grupo. Junto com os AIS, que serviram primariamente como tradutores, eles foram vistos como atores importantes na interação com os membros não indígenas das equipes e com os Yanomámi, especialmente como facilitadores dos serviços da EMSI 22. Porém, com o fim do convênio em 2004, os microscopistas não foram mais contratados, descontinuando a participação dos indígenas no combate à malária e nos processos de capacitação. Uma consequência foi o recrudescimento da doença. A capacitação dos AIS foi retomada em 2005, tratando de tópicos como conhecimento e funcionamento do corpo, noções de doença e contaminação, dentre outros, que, ignorando as formações anteriores, repetiram conhecimentos já dominados por alguns agentes.

Rocha 36 narra situação semelhante de conteúdos repetidos nos cursos de formação e capacitação para os AIS Baniwa, o que causou insatisfação dos indígenas. Após a implantação do DSEI-Rio Negro em 2000, os cursos ofertados nos moldes preconizados pela FUNASA, em módulos temáticos com períodos de concentração e dispersão, ignoraram as experiências de formação e capacitação anteriores, conquanto os conteúdos fossem semelhantes. Desde 1990 até 2000, realizaram-se oito cursos de formação e capacitação por várias instituições. Após a distritalização, somente quatro cursos foram ofertados pela FUNASA em convênios com uma ONG indígena e outra instituição. Segundo o autor, até o ano de 2006 é possível destacar “... a falta de um projeto pedagógico, o tecnicismo dos conteúdos, o reinício eterno das iniciativas dos cursos e a dificuldade em contemplar a especificidade da questão indígena" 36 (p. 95).

No caso de Santa Catarina, o DSEI Interior Sul inicialmente promoveu dois cursos introdutórios, um em 2001 e outro em 2002. Entre os 21 AIS que atuavam na Terra Indígena Xapecó em 2004 33, nove deles participaram dos cursos, mas somente dois fizeram ambos. Na Terra Indígena Laklãnõ, entre os sete AIS que trabalhavam entre fevereiro e outubro de 2004, três cursaram a capacitação de 2001. Em 2006, usando a proposta do DESAI/FUNASA de seis módulos, foram realizados dois deles no oeste de Santa Catarina. Na pesquisa de 2004, os AIS que participaram das capacitações não referiram espontaneamente tais cursos, e disseram não se lembrar daqueles de 2001 e 2002. Contudo, esses e outros agentes citaram capacitações anteriores aos DSEI que trataram sobre o uso de plantas medicinais e visita domiciliar, oferecidas de maneira esporádica. Os agentes recém-contratados afirmaram que não receberam cursos de capacitação no início de suas atividades, tendo recebido apenas orientações quanto a uma agenda de atividades e uma breve explicação sobre a visita domiciliar. Frente à falta de capacitação contínua, o aprendizado dos novos AIS sobre o preenchimento dos formulários se dava com a ajuda dos colegas mais antigos ou eventualmente nas reuniões com a enfermeira da EMSI.

Novo 30 também descreve a articulação, comunicação e troca de experiências entre os próprios AIS como forma de aprendizado e, inclusive, como fonte de estímulo para ingresso de outros indígenas nessa atividade. Entre os AIS do Parque Indígena do Xingu, valoriza-se essa forma de aprendizado, dado que é marcada pelo 
conhecimento adquirido na prática, “o único jeito de aprender" 30 (p. 117). É possível entender a valorização e o privilégio do aprendizado em serviço como repercussão das características e qualidade dos poucos cursos realizados.

A demanda por cursos de formação e palestras sobre temas específicos foi uma constante observada entre os Xokléng e os Kaingáng. A ausência de capacitações era indicada como motivo de insegurança no exercício cotidiano de suas práticas de atenção à saúde, assim como fonte de descrédito na eficácia de suas atividades e conselhos diante da população por eles atendida. Essa realidade estimulou vários AIS das duas Terras Indígenas a realizarem cursos de técnico ou auxiliar de enfermagem por esforço próprio, ou a terminarem o ensino médio com intenções de realizar tal curso. Ainda em Santa Catarina, por meio de uma solicitação de lideranças Kaingáng da Terra Indígena Xapecó à Universidade Federal de Santa Catarina, foi criado o "Projeto Pioneiro" em 2001, que formou auxiliares e técnicos de enfermagem, muitos deles atuando em suas aldeias 47 . O mesmo movimento atrás de melhores qualificações mediante cursos profissionalizantes, como de técnico ou auxiliar de enfermagem, foi destacado por Silva 32 entre os Atikum. A iniciativa dos AIS de buscar sua profissionalização fora das Terras Indígenas evidencia a necessidade do investimento na sua formação e, mais especificamente, evoca a reivindicação da certificação de nível técnico e do reconhecimento de sua profissão.

Como parte da centralização da FUNASA, em 2005 os cursos desenhados pelos seus funcionários de Brasília foram oficialmente lançados no formato de seis módulos intitulados Educação Profissional Básica para Agentes Indígenas de Saúde 48 , que trazem prioritariamente assuntos biomédicos e formação técnica. $\mathrm{O}$ primeiro módulo é uma introdução geral, e os restantes tratam da promoção da saúde e prevenção de parasitoses intestinais e doenças de pele; saúde do adulto e atenção às urgências; prevenção de doenças endêmicas; saúde da mulher, da criança e saúde bucal; e prevenção de DST/AIDS.

A proposta de formação da FUNASA por módulos mantém a perspectiva biomédica, desconsiderando as diferenças culturais, as especificidades epidemiológicas e a escolarização dos AIS a serem formados, bem como as dificuldades de cada DSEI (questões organizacionais, financeiras, de gestão etc.) para programar e executar tais módulos. O curso completo foi organizado para três anos, dividido em 12 etapas, sendo necessários, portanto, 258 módulos para os 34 DSEI. Analisando os módulos já ofertados, Raggio et al. 49 (p. 144) apontam que "em 2007, para o total de 3.833 agentes indígenas de saúde em curso de formação, foram concluídos 104 módulos, 40,3\% do total de 258 módulos programados. Foram realizadas 233 etapas do curso (sendo 129 concentrações e 104 dispersões), ou seja, 45,15\% de 516 no total. [...] A maioria dos DSEI tem encontrado dificuldades para a realização dos módulos, principalmente as etapas de dispersão, que exigem a permanência dos instrutores/supervisores pedagogicamente capacitados e o funcionamento regular dos serviços, haja vista a realização de 129 concentrações e apenas 104 dispersões em todos os DSEI com o curso de formação. O curso de formação foi concluído oficialmente nos DSEI Ceará, no segundo semestre de 2006, com uma turma de 14 AIS, e no DSEI Leste de Roraima, com a certificação de 372 AIS em abril de 2007".

Esses dados ainda fazem refletir sobre o perfil dos agentes que receberam as capacitações e sua permanência no trabalho, tendo em vista a problemática trazida pela alta rotatividade, discutida acima.

\section{Considerações finais}

A PNASPI 4 destaca a formação dos agentes indígenas de saúde como ponto central para promover uma atenção diferenciada, concebendo o agente como elo entre os saberes tradicionais e biomédicos. Nesta revisão, procuramos destacar que a definição do papel e a formação do AIS se iniciaram antes da implantação dos DSEI em localidades específicas e voltadas para a resolução de problemas particulares. Essas primeiras iniciativas envolveram atores engajados na proposta de atenção diferenciada, como antropólogos, profissionais de saúde, lideranças indígenas e outros, buscando, mediante processo pedagógico adequado, capacitar os indígenas para atividades em saúde, levando em conta as especificidades epidemiológicas e culturais.

A partir do Subsistema em 1999, os AIS foram formalmente incorporados ao modelo de atenção, tendo a FUNASA, em nível nacional, a responsabilidade de centralizar o processo de formação e capacitação. Essa centralização trouxe consequências nem sempre positivas, incluindo a desconsideração dos cursos anteriormente realizados, a proposição de módulos que predefinem os problemas de saúde, pautados pela agenda dos grandes programas do Ministério de Saúde, e as estratégias pedagógicas guiadas pelos modelos ocidentais de ensino-aprendizagem.

Embora boa parte dos estudos aqui analisados contemplarem as regiões Norte e Centrooeste do país, os dados obtidos pelas autoras em Santa Catarina podem ser ampliados para 
abranger o Sul e o Sudeste, considerando-se certa continuidade na organização e na estrutura dos serviços de saúde, pautadas na existência de dois grandes DSEI (Interior Sul e Litoral Sul).

Após a implantação dos DSEI, e apesar da proposta político-sanitária sobre o papel dos AIS, a revisão aponta que seu papel como mediador é marcado por conflitos e ambiguidades. A incorporação dos AIS na atenção primária é caracterizada, sobretudo, pela possibilidade de acesso e distribuição de medicamentos e pela função de comunicação com a comunidade. Apesar de ser definido como um representante da comunidade que participa dos serviços e atividades de controle social, eles, como membros contratados da equipe, percebem que a FUNASA é seu patrão e que são representantes de uma prática biomédica, criando uma situação de conflito de papéis. Para a comunidade, são representantes do serviço biomédico e para os demais profissionais da EMSI, membros com atividades voltadas ao preenchimento de planilhas, distribuição de medicamentos e comunicados relativos às questões de saúde. À medida que a falta de comunicação entre os AIS e o resto da equipe aumenta, essas ambiguidades e conflitos aparecem mais visivelmente.

Na rotina de trabalho, dada a dinâmica hierárquica das relações entre os profissionais da EMSI, a alta rotatividade e a descontinuidade dos cursos de capacitação, o AIS é indicado como o membro mais despreparado tecnicamente para atuar junto aos demais profissionais que, por sua vez, também reclamam da falta de capacitações para atuarem junto a populações indígenas. Ainda, notamos uma tensão entre a demanda da seleção dos AIS segundo os critérios da comunidade e os critérios técnicos exigidos pelo gestor.

Entendemos que não há discussão nem definição clara do conceito de atenção diferenciada. Nos documentos oficiais, atenção diferenciada é apresentada diversamente por noções de "articulação", "integração" e “incorporação” das práticas tradicionais. As noções de integração e incorporação submetem as práticas tradicionais à determinação de eficácia medida pela epistemologia biomédica, implicando uma fragmentação instrumental dos sistemas indígenas de saúde, selecionando somente aquelas práticas comprovadas cientificamente.

Também se torna evidente que o processo de trabalho segue estruturado segundo a lógica da organização de serviços de saúde pautados pelo modo capitalista de produção, pela racionalidade burocrática, pela hegemonia do saber biomédico e pela hierarquia de cargos legitimados por um sistema de treinamento formal. Nesse contexto, o AIS ocupa uma posição subalterna.
Além dessas ambiguidades e tensões, cumpre ressaltar que, desde a implantação dos DSEI, um processo contínuo de formação e capacitação não tem sido efetivado, e a falta e/ou descontinuidade dos cursos em quase todos os DSEI vem contribuindo de modo negativo para o reconhecimento e definição do papel do AIS. Por um lado, a escassez de cursos de capacitação para os agentes contribui para que os não indígenas das EMSI avaliem os AIS como técnicos desqualificados e, por outro, os AIS reclamam que não têm palestras nem cursos contínuos para ajudar a melhorar o atendimento dos problemas de saúde. Logo, a legitimidade frente à população indígena e frente aos membros não indígenas das EMSI tem sido um ponto relevante nas expectativas dos atores envolvidos na atenção à saúde, e os cursos de capacitação teriam um papel importante no Subsistema.

Como solução para a posição subalterna e a falta do reconhecimento da categoria de AIS, Garnelo et al. 50 citaram a implantação de um curso de formação de agentes comunitários indígenas de saúde no Alto Rio Negro, como resultado da mobilização inicial da articulação entre lideranças indígenas e AIS. Diversas instituições estão envolvidas nesse curso de formação com caráter técnico e profissionalizante, que propõe também elevar a escolaridade formal dos alunos. A proposta é formar e certificar 250 indígenas como agentes comunitários, categoria reconhecida pelo Ministério do Trabalho. Outra solução seria a formação em técnico de enfermagem, como nos contextos do Parque Indígena do Xingu e em Santa Catarina, com a diferença de que no primeiro caso a formação foi proposta pela conveniada com a FUNASA e, no segundo caso, os AIS buscaram a formação voluntariamente.

É nesse contexto, somado às reivindicações do movimento indígena e das Conferências Nacionais de Saúde Indígena, que destacamos a oficialização da ocupação dos AIS e agentes indígenas de saneamento (AISAN), que foram incluídos em 2010 como categoria profissional na família de "Agentes Comunitários de Saúde e Afins" da Classificação Brasileira de Ocupação do Ministério do Trabalho e Emprego 51. Em que pesem as diretrizes apontadas na PNASPI 4, até o momento o reconhecimento dos AIS e AISAN como profissionais de saúde, no que se refere à formação/capacitação, ao vínculo e aos seus papéis, ainda está sem resolução na prática, um desafio a ser superado pela recém-criada Secretaria Especial de Saúde Indígena 52, que assume toda a responsabilidade anteriormente definida para a FUNASA.

Positivamente, podemos apontar que a FUNASA se dedicou à contratação de AIS em 
número maior a cada ano. $\mathrm{O}$ aumento de contratações de AIS deve ser considerado no processo da implantação do Subsistema de Saúde Indígena. O AIS faz parte da reivindicação central das organizações indígenas na constituição da Política de Saúde para maior participação

\section{Resumo}

Os agentes indígenas de saúde (AIS), no Brasil, fazem parte de uma estratégia desenvolvida nas últimas duas décadas para a promoção de um modelo de atenção diferenciada à saúde indígena. Seu papel objetiva atender vários aspectos da política de atenção diferenciada, tais como o elo entre a equipe de saúde e a comunidade, e a mediação entre os saberes médico-científicos e os indígenas. Apesar do aumento significativo dos AIS nos últimos anos, falta ainda uma avaliação sobre suas competências e contribuições para a realização da atenção diferenciada. Este artigo, baseado em pesquisas realizadas pelas autoras e em uma revisão bibliográfica, analisa o papel do AIS por meio da sua incorporação, formação e participação nas equipes de saúde em diferentes contextos no país. Em que pese a importância dada ao papel do AIS, esta análise demonstra um conjunto de ambiguidades e contradições que dificulta o seu desempenho e uma contribuição maior para a atenção diferenciada.

Saúde de Populações Indígenas; Agentes Comunitários de Saúde; Serviços de Saúde do Indígena dos indígenas nos serviços, sendo também um importante recurso de renda e prestígio nos processos sociais e políticos comunitários. Aliás, como foi destacado nesta revisão, em muitas situações o AIS é o único profissional da EMSI presente na aldeia.

\section{Colaboradores}

As autoras participaram de todas as etapas do processo, desde a pesquisa empírica até a redação final do artigo.

\section{Agradecimentos}

Dedicamos esse artigo aos Kaingáng e Xokléng de Santa Catarina, que têm permitido ao longo das últimas duas décadas as pesquisas sobre saúde nas suas Terras Indígenas. Aos financiadores: Fundação de Amparo à Pesquisa e Inovação do Estado de Santa Catarina, Fundação de Amparo à Pesquisa do Estado do Amazonas, Conselho Nacional de Desenvolvimento Científico e Tecnológico, Ministério da Saúde (Secretaria de Ciência, Tecnologia e Insumos Estratégicos; Fundação Nacional de Saúde) e Organização Pan-Americana da Saúde. 


\section{Referências}

1. Declaration of Alma-Ata. International Conference on Primary Health Care; 1978, Sept 6-12; Alma-Ata, USSR. http://www.who.int/publications/almaata declaration_en.pdf (acessado em 21/Set/2009).

2. Constituição da República Federativa do Brasil; 1988. http://www.planalto.gov.br/ccivil_03/con stituicao/constitui\%C3\%A7ao.htm (acessado em 01/Out/2008).

3. Organização Internacional do Trabalho. Convenção ${ }^{\circ} .169$ sobre povos indígenas e tribais e Resolução referente à ação da OIT. 2a Ed. Brasília: Organização Internacional do Trabalho; 2005.

4. Fundação Nacional de Saúde. Política Nacional de Atenção à Saúde dos Povos Indígenas. 2a Ed. Brasília: Fundação Nacional de Saúde; 2002.

5. Brasil. Lei $n^{\circ}$. 10.507 , de 10 de julho de 2002. Cria a profissão de Agente Comunitário de Saúde e dá outras providências. Diário Oficial da União 2002; 11 jul.

6. Brasil. Lei no ${ }^{\circ}$ 11.350, de 5 de outubro de 2006, Regulamenta o § 5o do art. 198 da Constituição Federal, dispõe sobre o aproveitamento de pessoal amparado pelo parágrafo único do art. 2o da Emenda Constitucional no 51, de 14 de fevereiro de 2006, e dá outras providências. Diário Oficial da União 2006; 6 out.

7. Morosini MVGC. A política de formação dos agentes comunitários de saúde: memória de uma formulação em disputa nos anos 2003-2005 [Dissertação de Mestrado]. Rio de Janeiro: Instituto de Medicina Social, Universidade do Estado do Rio de Janeiro; 2009.

8. Krenak A, Gita de Oliveira A, Costa AM, Paiva Ferreira MJCM, Pinto NRS, Serra O. Documento final da Conferência Nacional de Proteção à Saúde do Índio. http://dtr2004.saude.gov.br/dab/docs/ geral/1cnsi.pdf (acessado em 22/Dez/2009).

9. Fundação Nacional de Saúde. Formação de agentes indígenas de saúde. Proposta de diretrizes. Brasília: Fundação Nacional de Saúde; 1996.

10. Confalonieri UEC, Verani CL. Agentes indígenas de saúde. In: Confalonieri UEC, organizador. Saúde de populações indígenas: uma introdução para profissionais de saúde. Rio de Janeiro: Escola Nacional de Saúde Pública, Fundação Oswaldo Cruz; 1993. p. 45-51.

11. Araújo M. Do corpo à alma. Missionários da Consolato e Índios Macuxi em Roraima. São Paulo: Fundação de Amparo à Pesquisa do Estado de São Paulo/Humanitas; 2006.

12. Mendonça SBM. O agente indígena de saúde no Parque Indígena do Xingu [Dissertação de Mestrado]. São Paulo: Pontifícia Universidade Católica de São Paulo; 1996.

13. Mendonça SBM. O agente indígena de saúde do Parque Indígena do Xingu: reflexões. In: Baruzzi $\mathrm{R}$, Junqueira $\mathrm{C}$, organizadores. Parque Indígena do Xingu: saúde, cultura e história. São Paulo: Universidade Federal de São Paulo/Terra Virgem; 2005. p. 227-46.
14. Oliveira LSS. Formação e inserção institucional de agentes indígenas de saúde no Sistema Único de Saúde: um estudo de possibilidades [Tese de Doutorado]. São Paulo: Universidade de São Paulo; 2002.

15. Oliveira LSS. O agente indígena de saúde do Parque Indígena do Xingu: perspectivas de formação e trabalho. In: Baruzzi R, Junqueira C, organizadores. Parque Indígena do Xingu: saúde, cultura e história. São Paulo: Universidade Federal de São Paulo/Terra Virgem; 2005. p. 247-58.

16. Bittencourt M, Toledo ME. Acre (1989-1994). In: Bittencourt M, Toledo ME, Argentino S, Oliveira LSS, organizadores. Acre, Rio Negro e Xingu: a formação indígena para o trabalho em saúde. São Paulo: Saúde sem Limites; 2005. p. 27-59.

17. Garnelo L. Projeto Rede Autônoma de Saúde Indígena: uma experiência de educação e comunicação em saúde indígena. In: Vasconcelos EM, organizador. A saúde nas palavras e nos gestos: reflexões da Rede Educação Popular e Saúde. São Paulo: Editora Hucitec; 2001. p. 237-60.

18. Argentino S. Rio Negro (1996-2000). In: Bittencourt M, Toledo ME, Argentino S, Oliveira LSS, organizadores. Acre, Rio Negro e Xingu: a formação indígena para o trabalho em saúde. São Paulo: Saúde sem Limites; 2005. p. 82-6.

19. Erthal RMC. A formação do agente de saúde indígena Tikúna no Alto Solimões: uma avaliação crítica. In: Coimbra Jr. CEA, Santos RV, Escobar AL, organizadores. Epidemiologia e saúde dos povos indígenas no Brasil. Rio de Janeiro: Editora Fiocruz; 2003. p. 197-216.

20. Diehl EE. Entendimentos, práticas e contextos sociopolíticos do uso de medicamentos entre os Kaingáng (Terra Indígena Xapecó, Santa Catarina, Brasil) [Tese de Doutorado]. Rio de Janeiro: Escola Nacional de Saúde Pública, Fundação Oswaldo Cruz; 2001.

21. Souza NMSF, Calvacante MS, Brandão MC. Agentes de saúde indígena: realidades pluriétnicas e experiências de formadores. In: Brandão MC, Paula NC, Athias R, organizadores. Saúde indígena em São Gabriel da Cachoeira (AM): uma abordagem antropológica. Recife: Liber; 2002. p. 51-72.

22. Bissera RS. Ainda estamos vivos: uma etnografia da saúde Sanumá [Tese de Doutorado]. Brasília: Programa de Pós-graduação em Antropologia, Universidade de Brasília; 2006.

23. Oliveira MC. Os curadores Kaingáng e a recriação de suas práticas: estudo de caso na aldeia Xapecó (Oeste de S.C.) [Dissertação de Mestrado]. Florianópolis: Programa de Pós-graduação em Antropologia Social, Universidade Federal de Santa Catarina; 1996.

24. Bittencourt M, Toledo M, Argentino S, Oliveira LSS, organizadores. Acre, Rio Negro e Xingu: a formação indígena para o trabalho em saúde. São Paulo: Saúde sem Limites; 2005. 
25. Garnelo L. Poder, hierarquia e reciprocidade: saúde e harmonia entre os Baniwa do Alto Rio Negro. Rio de Janeiro: Editora Fiocruz; 2003.

26. Ministério da Saúde. Portaria no ${ }^{\circ} 2.656$ de 17 de outubro de 2007. Dispõe sobre as responsabilidades na prestação da atenção à saúde dos povos indígenas, no Ministério da Saúde e regulamentação dos Incentivos de Atenção Básica e Especializada aos Povos Indígenas. Diário Oficial da União 2007; 18 out.

27. Coordenação de Saúde do Índio, Departamento de Operações, Fundação Nacional de Saúde. Formação de agentes indígenas de saúde. Brasília: Fundação Nacional de Saúde; 1999.

28. Brasil. Lei no. 9 394, de 20 de dezembro de 1996. Estabelece as diretrizes e bases da educação nacional. Diário Oficial da República Federativa do Brasil 1996; 23 dez.

29. Conselho Nacional de Saúde. III Conferência Nacional de Saúde Indígena - relatório final. http:// dtr2004.saude.gov.br/dab/docs/geral/3cnsi.pdf (acessado em 31/Dez/2009).

30. Novo MP. Os agentes indígenas de saúde no Alto Xingu [Dissertação de Mestrado]. São Carlos: Programa de Pós-graduação em Antropologia, Universidade Federal de São Carlos; 2008.

31. Dias-Scopel RP, Langdon EJ, Scopel D. Expectativas emergentes: a inserção do agente indígena de saúde Xokleng na equipe multidisciplinar de atenção à saúde indígena. Tellus 2007; 13:51-73.

32. Silva G. "Chama os Atikum que eles Desatam Já": práticas terapêuticas, sabedores e poder [Dissertação de Mestrado]. Recife: Programa de Pós-graduação em Antropologia, Universidade Federal de Pernambuco; 2007.

33. Langdon EJ, Diehl EE, Wiik FB, Dias-Scopel R. A participação dos agentes indígenas de saúde nos serviços de atenção à saúde: a experiência em Santa Catarina, Brasil. Cad Saúde Pública 2006; 22:2637-46.

34. Garnelo L, Macedo G, Brandão LC. Os povos indígenas e a construção das políticas de saúde no Brasil. Brasília: Organização Pan-Americana da Saúde; 2003.

35. Novo MP. Os agentes indígenas de saúde do Alto Xingu. Brasília: Paralelo 15/Associação Brasileira de Antropologia; 2010.

36. Rocha E. Uma etnografia das práticas sanitárias no Distrito Sanitário Especial Indígena do Rio Negro Noroeste do Amazonas [Dissertação de Mestrado]. Manaus: Programa de Pós-graduação em Sociedade e Cultural na Amazônia, Universidade Federal das Amazonas; 2007.

37. Rocha CC. Adoecer e curar: processos da sociabilidade Kaingáng [Dissertação de Mestrado]. Florianópolis: Programa de Pós-graduação em Antropologia Social, Universidade Federal de Santa Catarina; 2005.

38. Fundação Nacional de Saúde. Agentes indígenas de saúde. http://www.funasa.gov.br/Web\%20 Funasa/ind/ind01.htm\#2 (acessado em 29/Dez/ 2009).
39. Wawzyniak JV. Assombro de olhada de bicho: uma etnografia das concepções e ações em saúde entre Ribeirinhos do Rio Tapajós, Pará - Brasil [Tese de Doutorado]. São Carlos: Programa de Pós-graduação em Antropologia, Universidade Federal de São Carlos; 2008.

40. Dias-Scopel RP. O agente indígena de saúde Xokleng - uma abordagem da antropologia da saúde [Dissertação de Mestrado]. Florianópolis: Programa de Pós-graduação em Antropologia Social, Universidade Federal de Santa Catarina; 2005.

41. Marinho GL, Ott AT. Agentes indígenas de saúde de Rondônia: um estudo etnográfico (relatório preliminar). Porto Velho: Programa Institucional de Bolsas de Iniciação Científica, Universidade de Rondônia/Centro de Estudos de Saúde Indígena de Rondônia/CNPq; 2007.

42. Scopel D. Saúde e doença entre os índios Mura de Autazes (Amazonas): processos socioculturais e a práxis da autoatenção [Dissertação de Mestrado]. Florianópolis: Programa de Pós-graduação em Antropologia Social, Universidade Federal de Santa Catarina; 2007.

43. Imbiriba EB, Basta PC, Pereira ES, Levino A, Garnelo L. Hanseníase em populações indígenas do Amazonas, Brasil: um estudo epidemiológico nos municípios de Autazes, Eirunepé e São Gabriel da Cachoeira (2000 a 2005). Cad Saúde Pública 2009; 25:972-84.

44. Vianna JJB, Ott AMT. Agentes indígenas de saúde no DSEI Porto Velho: um estudo etnográfico. Pesquisa e Criação 2007; 5. http://www.pibic.unir.br/ (acessado em 27/Dez/2009).

45. Langdon EJ, Diehl EE. Participação e autonomia nos espaços interculturais de saúde indígena: reflexões a partir do sul do Brasil. Saúde Soc 2007; 16:19-36.

46. Teixeira CC. Fundação Nacional de Saúde: a política brasileira de saúde indígena vista através de um museu. Etnográfica 2008; 12:323-51.

47. Follmann HBC. Indígenas como trabalhadores da enfermagem: o papel de técnicos e auxiliares no modelo de atenção à saúde indígena [Dissertação de Mestrado]. Florianópolis: Programa de Pós-graduação em Saúde Coletiva, Universidade Federal de Santa Catarina; 2011.

48. Fundação Nacional de Saúde. Educação profissional básica para agentes indígenas de saúde. http:// www.funasa.gov.br/internet/Bibli_saudeInd.asp (acessado em 02/Jan/2010).

49. Raggio A, Pinto N, Morais P. Análise da gestão. In: Institute of Development Studies/Saúde Sem Limites/Centro Brasileiro de Análise e Planejamento, organizadores. Diagnóstico situacional do Subsistema de Saúde Indígena - relatório inicial (revisado). Brasília: Consórcio Institute of Development Studies/Saúde Sem Limites/Centro Brasileiro de Análise e Planejamento; 2009. p. 112-50.

50. Garnelo L, Rocha E, Peiter P, Sampaio S, Santos E, Pontes AL, et al. Formação técnica de agente comunitário indígena de saúde: uma experiência em construção no Rio Negro. Trab Educ Saúde 2009; 7:373-85. 
51. Secretaria de Políticas Públicas de Emprego, Ministério do Trabalho e Emprego. Classificação Brasileira de Ocupações - códigos, títulos e descrições. 3a Ed. Brasília: Ministério do Trabalho e Emprego; 2010.

52. Brasil. Decreto no. 7.336, de 19 de outubro de 2010. Aprova a estrutura regimental e o quadro demonstrativo dos cargos em comissão e das funções gratificadas do Ministério da Saúde, e dá outras providências. Diário Oficial da União 2010; 20 out.

53. Fundação Nacional da Saúde. Relatório das principais atividades e resultados - 1999-2002. http:// www.funasa.gov.br/sitefunasa/fns/pdf/relatorio_ 1999-2002 (acessado em 13/Mai/2005).
54. Fundação Nacional de Saúde. Programa de formação para agentes indígenas de saúde. http:// www.neppi.org/anais/textos/pdf/programa_for macao_agentes_indigenas.pdf (acessado em 08/ Out/2009).

55. Institute of Development Studies/Saúde Sem Limites/Centro Brasileiro de Análise e Planejamento. Diagnóstico Situacional do Subsistema de Saúde Indígena - relatório inicial (revisado). Brasília: Consórcio Institute of Development Studies/Saúde Sem Limites/Centro Brasileiro de Análise e Planejamento; 2009.

Recebido em 12/Dez/2011

Versão final reapresentada em 11/Mar/2012

Aprovado em 15/Mar/2012 